\title{
OPEN Dyeing of cotton fabric materials with biogenic gold nanoparticles
}

\author{
M. Sivakavinesan ${ }^{\bowtie}$, M. Vanaja \& G. Annadurai
}

The present work aimed at synthesizing gold nanoparticles in a biological method employing fruit peel waste dumped in the environment. The peels of Garcinia mangostana (Mangostan), were collected from the nearby tourist spot during the season. The collected fruit peels were washed, dried, powder and extracted by using boiling water and acetone. The precipitated extract was dried and powdered for further use. The dried and powdered peel extract was added to the gold solution and boiled to $80^{\circ} \mathrm{C}$ and the color change is observed. The color change indicates the completion of the synthesis of gold nanoparticles. The effect of $\mathrm{pH}$, gold ion concentration, peel extract powder concentration, and the temperature was tested by varying the parameters. The biosynthesized nanoparticles were characterized using the UV-Vis spectrophotometer to identify the surface plasmon resonance peaks corresponding to gold nanoparticles. The bio-moieties responsible for the synthesis of gold nanoparticles were identified using the Fourier Transform Infra-Red Spectroscopy. The crystalline nature was detected by using an X-Ray Diffractometer. Atomic Force Microscope viewed the 3D surface image of the gold nanoparticle. The shape and morphology of the nanoparticle were identified by using a Field Emission Scanning Electron Microscope. The active compounds for gold nanoparticle synthesis were identified using Gas Chromatography-Mass Spectrometry. The gold nanoparticle was synthesized in various colors and used for dyeing cotton fabrics. The dyed cotton materials were exposed to various stress conditions to determine the color fastening.

Mangostan (Garcinia mangostana Linn.) (Clusiaceae) is a tropical tree distributed in India, Myanmar, Malaysia, Philippines, Sri Lanka, and Thailand. G. mangostana grows up to 6-25 $\mathrm{m}^{1}$. The fruits of G. mangostana show good taste hence it is called as "queen of fruits" and it contains a rich source of phenolic compounds such as xanthones, condensed tannins, and anthocyanins ${ }^{2,3}$. In Ayurvedic medicine, the pericarp of mangostan-fruit has been widely used against inflammation, diarrhea, cholera, and dysenter $y^{4,5}$.

Nanotechnology is an extremely powerful emerging technology that is expected to have a substantial impact on technology now and in the future ${ }^{6}$. Before 100 years, Michael Faraday reported the colloidal form of gold nanoparticles. Gold nanoparticles are one of the most extensively studied nanomaterials ${ }^{7}$ and were synthesized through several biomaterials namely Volvariella volvacea (Singer) ${ }^{8}$, eggshell membrane ${ }^{9}$, Stoechospermum marginatum (Agardh) ${ }^{10}$, Solanum nigrum L ${ }^{11}$, Trichoderma harzianum (Rifai) ${ }^{12}$, Brassica oleracea L. ${ }^{13}$, Hibiscus sabdariffa L. ${ }^{14}$, Couroupita guianensis (Aubl) fruit extract ${ }^{15}$, Elaeis guineensis (Jacq) ${ }^{16}$ and Crocin ${ }^{17}$. The analysis of surface plasmon resonance absorption band can also provide valuable information on the size, structure, and aggregation properties of gold nanoparticles. The dielectric constant of the surrounding medium determines the shape and particle size of the nanoparticle ${ }^{18}$. Gold nanoparticles are employed for various applications including functionalization of bamboo pulp ${ }^{19}$, sensors ${ }^{20,21}$, digital decoration ${ }^{22}$, antioxidants ${ }^{15}$, anti-cancer ${ }^{17}$, catalytic degradation of dyes ${ }^{23,24}$, and colourants ${ }^{18}$.

The dyes disposed of in textiles, cosmetics, food, leather, and plastic industries cause major environmental pollutants which disrupt and cause various toxic effects like mutagenic or carcinogenic in aquatic organisms. The dyes can also accumulate in soil and it takes a higher half-life time for degradation ${ }^{23}$. These negative impacts on the environment make an alarm to research communities and find an alternate solution for dyeing. Moreover, textile products with enriched functionalities like antibacterial, antistatic, stain-resistant, and UV protection are being demanded by consumers ${ }^{25}$.

The surface plasmon resonance property of nano solutions (Gold, silver, and copper) was used for coloring purposes $^{22}$. Metal nanoparticles represent noble bright colors due to the localized surface plasmon resonance. The different colors of noble metal nanoparticles have been used to dye cotton and wool ${ }^{26}$. Nanocrystalline cobalt aluminate spinel was considered as a nano pigment that contains blue color enhanced by the addition of starch solution $^{27}$. Afshari et al. ${ }^{28}$ reported that dyeing of polyester fabric using copper nanoparticles and assessed its 
antimicrobial property. In the present study, gold nanoparticles were synthesized through a greener method and it was applied for the dyeing process.

\section{Materials and methods}

Preparation of fruit peel extract powder. The fruit peels of G. mangostana were collected from Courtal$\operatorname{lam}\left(8.9342^{\circ} \mathrm{N} 77.2778^{\circ} \mathrm{E}\right)$, Tirunelveli, Tamilnadu, India. Collected fruit peels were washed with distilled water and dried in shade to remove adhering dirt and dust. The fruit peels $(100 \mathrm{~g})$ were boiled in distilled water at $90^{\circ} \mathrm{C}$ for $30 \mathrm{~min}$ and crushed by adding $100 \mathrm{ml}$ of distilled water and the resultant extract was filtered through a clean muslin cloth. Equal volumes of chilled acetone were added to filter the precipitate. The resulting precipitate was collected by centrifugation (Eltek RC4100F) at $7000 \mathrm{rpm}$ for $10 \mathrm{~min}$, air-dried in a hot air oven, powdered form, and used for further experiments.

Green synthesis of gold nanoparticles. Chloroauric acid $\left(\mathrm{HAuCl}_{4}\right)$ in double-distilled water $(1 \mathrm{mM})$ was used as a source of gold nanoparticles throughout the experiments. The reaction mixture contains $10 \mathrm{mg}$ fruit peel extract powder, $2 \mathrm{ml}$ chloroauric acid solution $(1 \mathrm{mM})$. The reaction mixtures were heated at $80^{\circ} \mathrm{C}$ in a water bath for $3 \mathrm{~min}$. These were monitored for different time intervals and the nanoparticles and microstructures formed were characterized further.

Synthesis of different colours of gold nanoparticle solution. Gold nanoparticles were synthesized in different colors for dyeing applications. The color of the solution depends on the surface plasmon resonance effect (SPR) of the gold nanoparticle synthesized. The different colors of solution were prepared by increasing the concentration of chloroauric acid solution from $10^{-2}, 10^{-3}, 10^{-4}, 10^{-5}$, and $10^{-6} \mathrm{M}$.

Characterization of nanoparticles. The reduction of gold ions was monitored by using a double beam UV-vis spectrophotometer (Lambda 25, Perkin Elmer, Singapore) of the reaction medium in the wavelength range of 450-700 $\mathrm{nm}$ with a $10 \mathrm{~mm}$ quartz cell. FTIR confirmed functional biomolecules associated with the reduction of gold ions into gold nanoparticles (Spectrum two, Perkin Elmer, Singapore). Biosynthesized gold nanoparticles were centrifuged at $7000 \mathrm{rpm}$ for $15 \mathrm{~min}$ and the pellets were washed with distilled water. The centrifuging and re-dispersing process were repeated thrice. The samples were dried and analyzed at a wave region of $400-4000 \mathrm{~cm}^{-1}$.

The purified gold nanoparticle structures and compositions were analyzed by XRD (Panalytical X 'Pert Powder X' Celerator Diffractometer). The crystalline nature of the nanoparticles was analyzed at the $2 \theta$ ranges of $10-80^{\circ}$. The morphology and size of the gold nanoparticles were found by Field Emission Scanning Electron Microscope (Philip model CM 200). Atomic Force Microscopy analysis (Nanosurf EasyScan 2) was done to examine the shape and size of the nanoparticle synthesized using the biological technique.

The mangostan peel extract was subjected to Gas Chromatography-Mass Spectrometry (GC-MS) (Perkin Elmer, Clarus 680) analysis to identify the compounds responsible for the synthesis of gold nanoparticles. The acquisition parameters for GC-MS are, Oven: Initial temp $60^{\circ} \mathrm{C}$ for $2.80 \mathrm{~min}$, ramp $10^{\circ} \mathrm{C} / \mathrm{min}$ to $300^{\circ} \mathrm{C}$, hold $6 \mathrm{~min}$, InjA auto $=260^{\circ} \mathrm{C}$, Volume $=1 \mu \mathrm{L}$, Split $=10: 1$, Carrier Gas $=\mathrm{He}$, Solvent Delay $=2.80 \mathrm{~min}$, Transfer Temp $=230{ }^{\circ} \mathrm{C}$, Source Temp $=230^{\circ} \mathrm{C}$, Scan: 40 to $600 \mathrm{Da}$, Column $30 \times 250 \mu \mathrm{m}$. Further, the gold nanoparticles coated cotton fabrics were analyzed for the presence of nanoparticles using a Scanning Electron Microscope (Carl Zeiss, Model EVO 18).

Gold nanoparticle as dye. Thermofixing method. The different colored gold nanoparticles were fixed on the wicks (cotton with length $10 \mathrm{~cm}$ ) material by thermo fixing method. The wicks pieces were dyed by boiling with gold nanoparticle solution at a higher temperature range from 120 to $160^{\circ} \mathrm{C}$. Initially, the dyed wicks were kept at $120^{\circ} \mathrm{C}$ for $15 \mathrm{~min}$ without alteration in temperature. After $15 \mathrm{~min}$, the temperature of boiling was increased to $160^{\circ} \mathrm{C}$ and kept for $1 \mathrm{~h}$. Fabric materials were rinsed with water and then dried Material was again rinsed well after reduction cleaning and then dried in the $\operatorname{air}^{29}$.

Colour fastness of the fabrics. The Thermo fixed wick pieces were tested for colorfastness against drastic conditions. Each dyed fabric piece was dried at $45^{\circ} \mathrm{C}$ for $24 \mathrm{~h}$ and tested for colorfastness against different situations. The color fastness of the colored fabrics was verified by dipping the fabrics in commercial detergent solutions for $24 \mathrm{~h}$ and by scouring. Following this process, the pieces were also treated with acid ( $1 \%$ acetic acid), alkali ( $1 \%$ $\mathrm{KOH})$, and hot water with commercial detergent $(\mathrm{pH} \sim 11)$ for $1 \mathrm{~h}$. The color fastnesses of the fabrics were also determined by the exposure to sunlight during summer (April-May; average atmospheric temperature $36^{\circ} \mathrm{C}$ ) at different time scales, i.e. 1-10 days.

\section{Results and discussion}

Green synthesis and characterization of gold nanoparticles. Visual observation. Gold nanoparticle formation was primarily identified by color change visually. The observation of color changes, from colorless solution into purple or pink color, was one of the processes to identify the synthesis of gold nanoparticles in the solution (Fig. 1). The peel powder extract was exposed to gold chloride solution and boiled, the green synthesis of nanoparticles started within few minutes and was identified by color change. Gold nanoparticles show different colors for each concentration of the chosen chloroauric acid solution. The color ranges from yellow, purple, and pink. The color arises because of the excitation of the surface plasmon resonance effect of gold nanoparticles. Previously, the report of Singaravelu et al. ${ }^{30}$ and Inbakandan et al. ${ }^{31}$ has also shown that the gold nanoparticle 

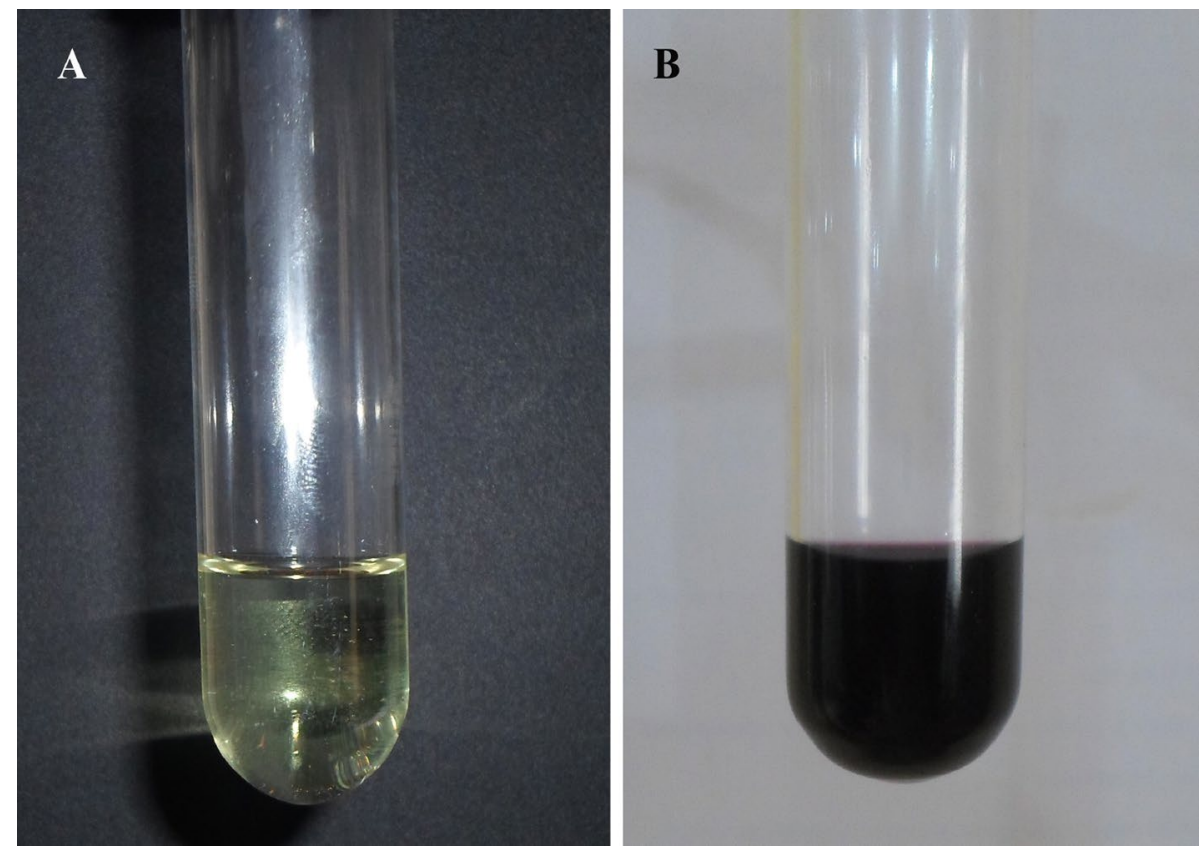

Figure 1. Gold solution (A) and gold nanoparticle (B) colloidal solution.

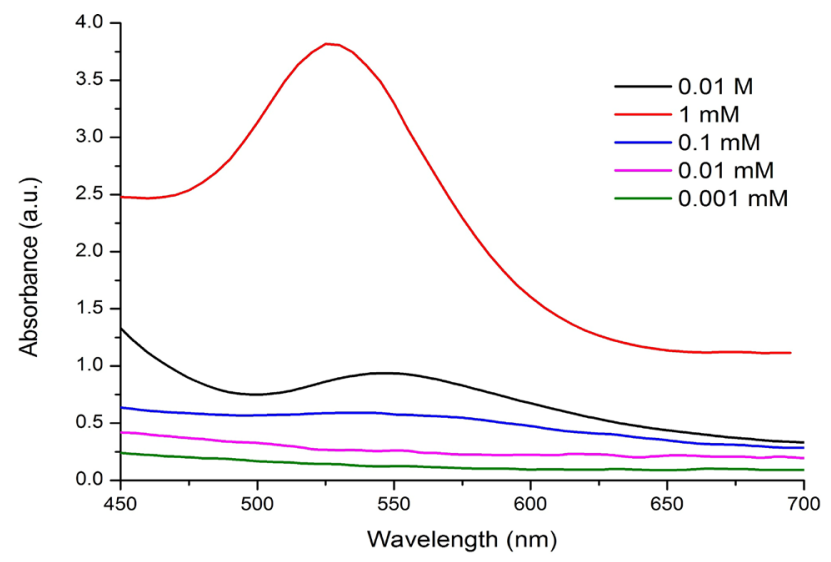

Figure 2. UV-Vis spectroscopy image of gold nanoparticle at various concentrations.

synthesis by using marine algae Sargassum wightii and the marine sponge Acanthella elongate, respectively. The result color change got in this investigation is very interesting in terms of the identification of potential plants for synthesizing the nanoparticles ${ }^{32}$.

UV-vis spectrophotometer analysis (Effect of gold ion concentrations). UV-vis spectroscopy is an important preliminary technique used to determine the formation of nanoparticles at different concentrations of gold ions. Figure 2 shows the effect of gold ion concentration in the gold nanoparticles synthesis process by using the peel powder of mangostan. Characteristic surface plasmon absorption bands are observed at $530 \mathrm{~nm}$ for the purplecolored gold nanoparticles synthesized from $1 \mathrm{mM}\left(10^{-3} \mathrm{M}\right)$ gold ion concentration. The sharp peak at $530 \mathrm{~nm}$ is the characteristic peak of monodispersed spherical nanoparticles. The $1 \mathrm{mM}$ concentration shows a narrow band with increased absorbance, whereas other concentrations like $10^{-4} \mathrm{M}$ show a broad peak at $547 \mathrm{~nm}$ with low absorbance. It reveals large-sized nanoparticles because of the higher multipole plasmon excitation ${ }^{33,34}$. Single SPR band formation with increased absorbance at lower wavelength reveals the synthesis of the small spherical shape of nanoparticles ${ }^{35}$.

$X$-ray diffraction analysis. The crystalline size and structure of the gold nanoparticles were determined by XRD in the entire spectrum of $2 \theta$ values ranging from 10 to $80^{\circ}$. Peel powder extract mediated synthesized gold nanoparticles using mangostan shows four strong diffraction peaks at the $2 \theta$ values of $38.30^{\circ}, 44.51^{\circ}, 64.82^{\circ}$ and $78.56^{\circ}$ (Fig. 3) could be assigned the plane of (llll), (2 $\left.\begin{array}{lll}2 & 0\end{array}\right),\left(\begin{array}{lll}2 & 2 & 0\end{array}\right)$ and (3 111$)$, respectively shows the gold 


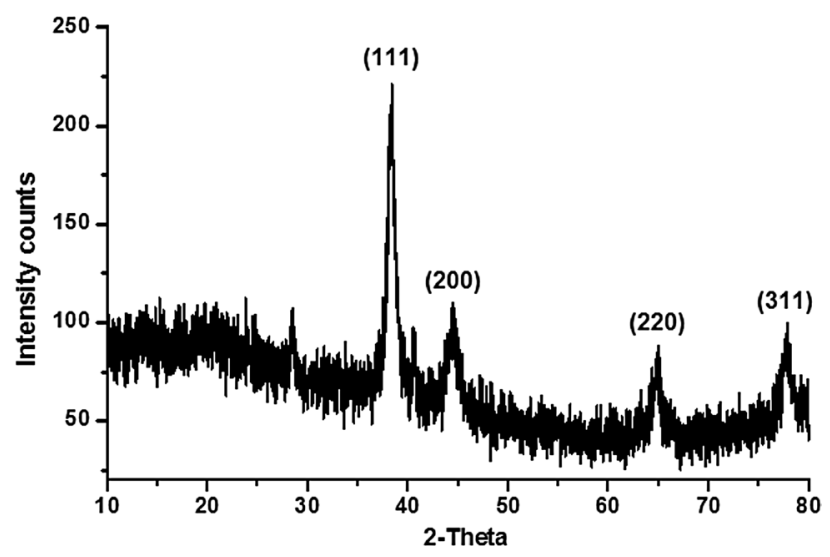

Figure 3. XRD pattern of gold nanoparticle.
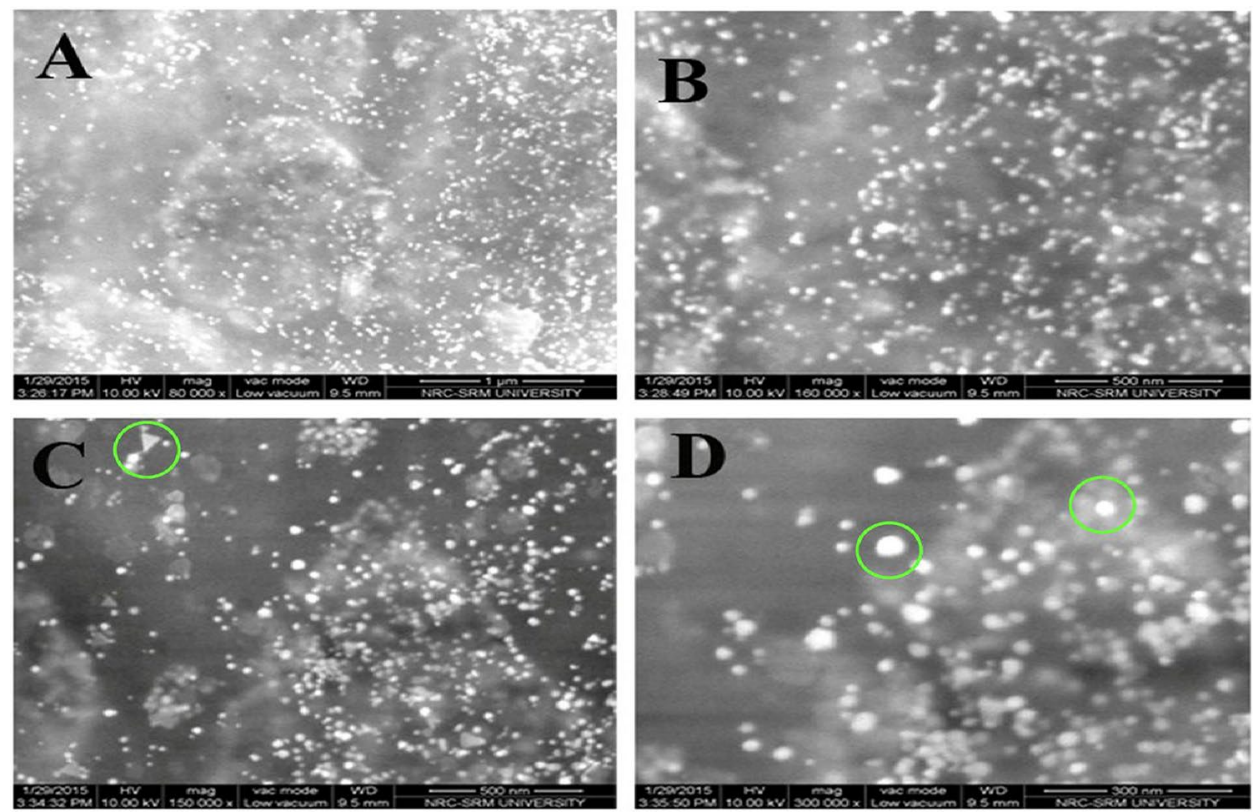

Figure 4. FESEM image of gold nanoparticles synthesized using mangostan peel extract (A) $1 \mu \mathrm{m}$, (B) $500 \mathrm{~nm}$, (C) $500 \mathrm{~nm},($ D) $300 \mathrm{~nm}$.

nanoparticles are face-centered cubic crystalline structure. The synthesized gold nanoparticles are compared with standard gold and pure nanogold particles which are published by Joint Committee on Powder Diffraction Standards (File nos. 04-0783 and 84-0713). A comparison of our XRD spectrum with the standard confirmed that the gold nanoparticles formed in our experiments were nanocrystals. The mean size of synthesized gold nanoparticles was calculated using Debye-Scherrer's Eq. ${ }^{36}$ by determining the width of the (lll 11 ) Bragg reflection using the following equation.

$$
\mathrm{D}=\mathrm{K} \lambda / \beta \cos \theta
$$

where $\mathrm{D}$ is the average crystallite domain size perpendicular to the reflecting planes, $\lambda$ is the $\mathrm{X}$-ray wavelength $(1.5418 \AA$ A), $\beta$ is the width of the XRD peak at half-height, $\theta$ is the diffraction angle, and $\mathrm{K}$ is the Scherrer coefficient (0.89) shape factor for spherical particles. The calculated average particle size of the synthesized nano gold using mangostan peel powder extract was found to be $128 \mathrm{~nm}$. The present report is well-matched with the standard gold and the report of Singaravelu et al. ${ }^{30}$ and Shukla et al. ${ }^{37}$.

Field emission scanning electron microscope. The surface morphology of the synthesized gold nanoparticles was identified on the nanoscale bar by Field Emission Scanning Electron Microscope. FESEM image had shown the shape and distribution of the gold nanoparticles synthesized by using peel extract of mangostan (Fig. 4). FESEM micrographs show synthesized nanoparticles were adsorbed on the surface of peel powder. FESEM image shows individually dispersed gold nanoparticles and several aggregates synthesized with irregular shapes using peel 

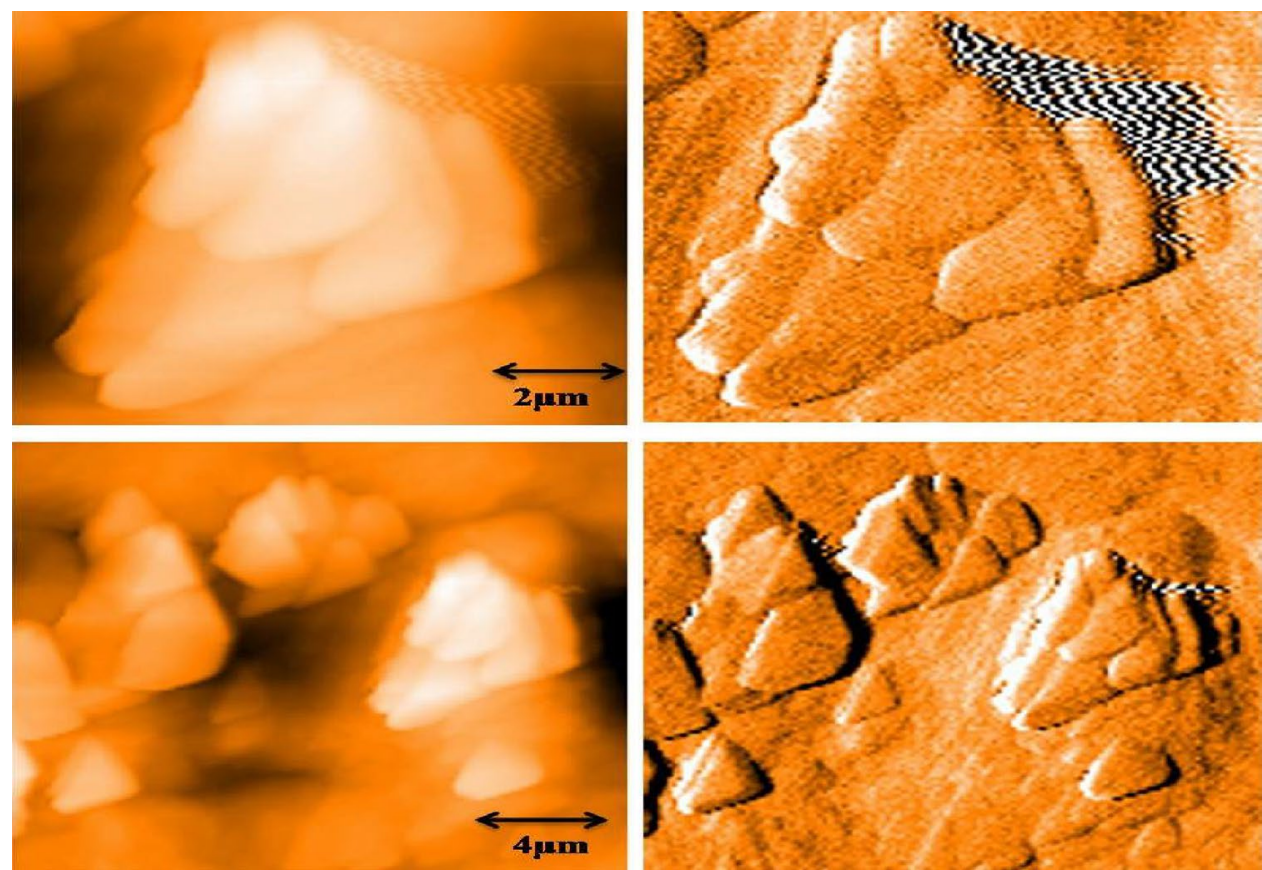

Figure 5. Atomic Force Microscope image of mangostan peel mediated synthesized gold nanoparticles at $2 \mu \mathrm{m}$ and $4 \mu \mathrm{m}$.

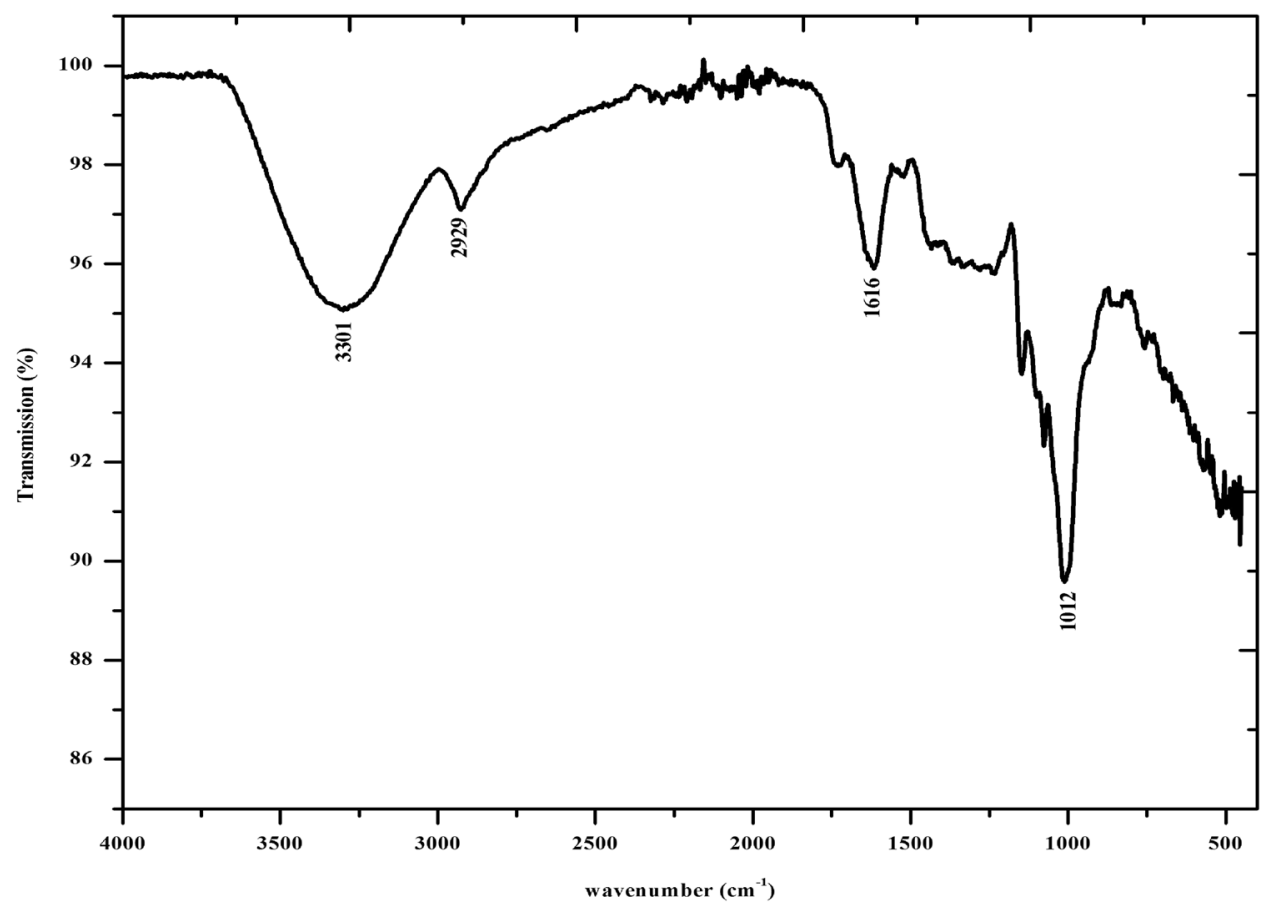

Figure 6. FTIR spectrum of gold nanoparticle.

\begin{tabular}{|l|l|l|}
\hline S. no. & Peak position & Corresponding functional groups \\
\hline 1 & 3301 & O-H stretch of alcohols, N-H stretch of $1^{\circ}, 2^{\circ}$ amines, amides \\
\hline 2 & 2929 & O-H stretch of carboxylic acids, C-H stretch of alkanes \\
\hline 3 & 1616 & N-H bend of $1^{\circ}$ amines \\
\hline 4 & 1012 & C-O stretch of carboxylic acids, alcohols, esters, ethers \\
\hline
\end{tabular}

Table 1. Peak position and corresponding functional groups present in gold nanoparticles synthesized using mangostan peel extract powder. 


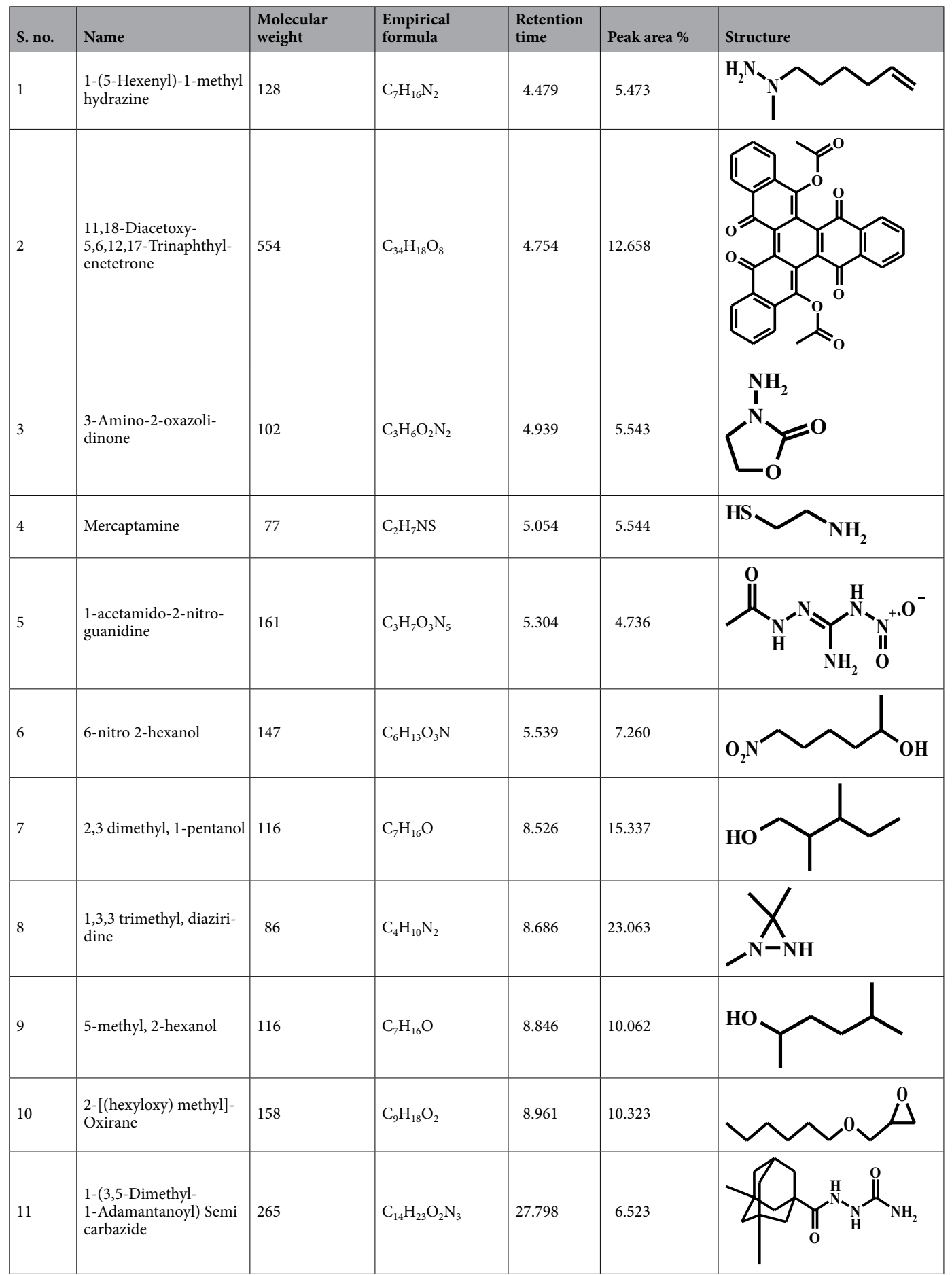

Table 2. GCMS profile of compounds present in mangostan peel extract powder.

powder extract of mangostan. It illustrates the particles are predominantly spherical and some particles aggregate into larger particles with no well-defined morphology and the size of the gold nanoparticles ranging from 75 to $130 \mathrm{~nm}$. The similar irregular shape of gold nanoparticles with different sizes from 5 to $100 \mathrm{~nm}$ was reported by using macerated clove bud's solution ${ }^{38}$.

Atomic force microscope. Atomic force microscopy (AFM) was also known as scanning force microscopy (SFM). AFM is a basic technique and inevitable for all nanoscopic research. The AFM image of gold nanoparticles synthesized using the peel extract powder of mangostan is shown in Fig. 5. The particles synthesized 


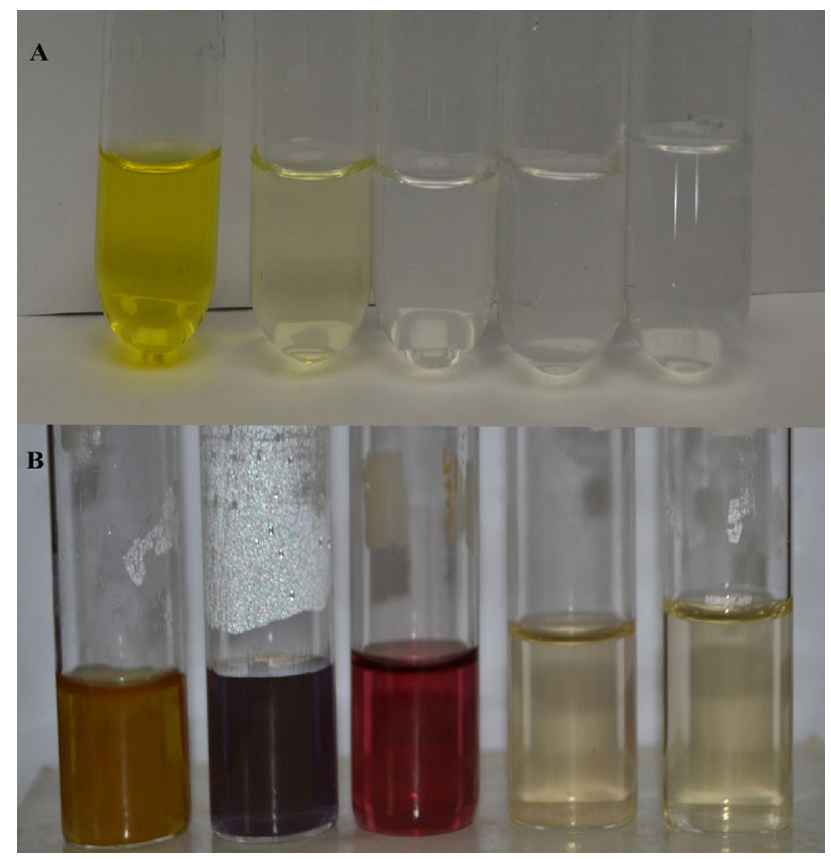

Figure 7. (A) Gold solution at various concentration, (B) gold nanoparticle colloid solutions.

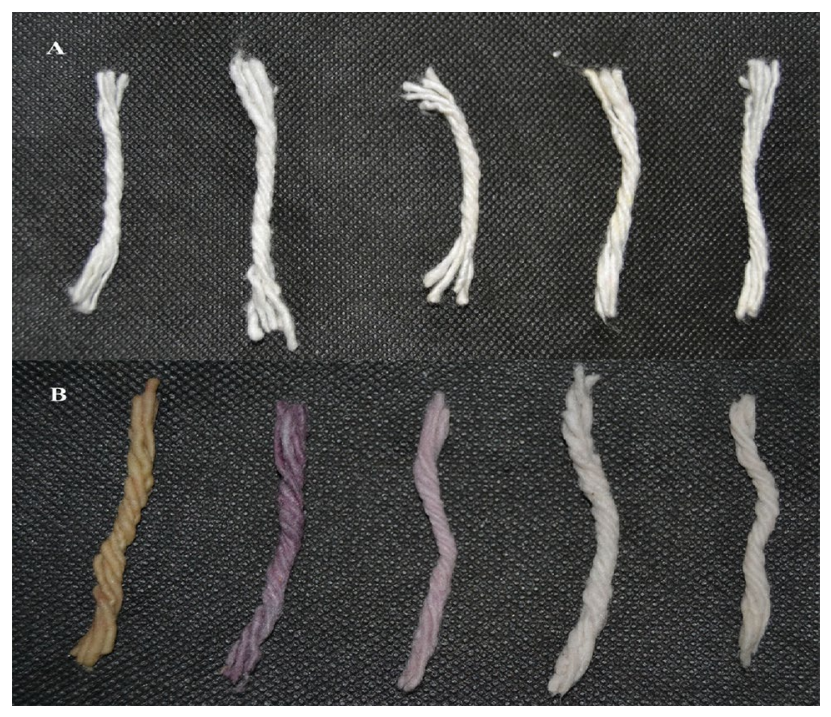

Figure 8. (A) cotton material before dyeing, (B) cotton material dyed using gold nanoparticle colloid solution.

agglomerated with each other to form a sheet-like form ${ }^{39}$. The particles were viewed at 2 and $4 \mu \mathrm{m}$ distances and also in a $3 \mathrm{D}$ pattern. The particles synthesized using mangostan formed layers and are smaller.

Fourier transform infra-red spectroscopy. The FTIR spectrum of gold nanoparticles synthesized using the peel extract powder of mangostan is presented in Fig. 6 . The functional groups responsible for the synthesis of nanoparticles are denoted by the corresponding peaks arising due to the infrared radiations. The functional groups corresponding to the peak in the gold nanoparticles are presented in Table 1.

Gas chromatography mass spectrum analysis. The GC-MS analysis revealed that eleven major constituents were present in the mangostan peel extract (Table 2). The compounds were first identified by Gas Chromatography and then the mass spectrum of the analyzed sample was matched with the NIST-2008 libraries. The analyses of mangostan peel extract indicate the presence of eleven major compounds with significant matches to 1, 3, 3 trimethyl, diaziridine (23.063\%) followed by 2, 3 dimethyl, 1-pentanol (15.337\%) and 11, 18-Diacetoxy-5, 6, 


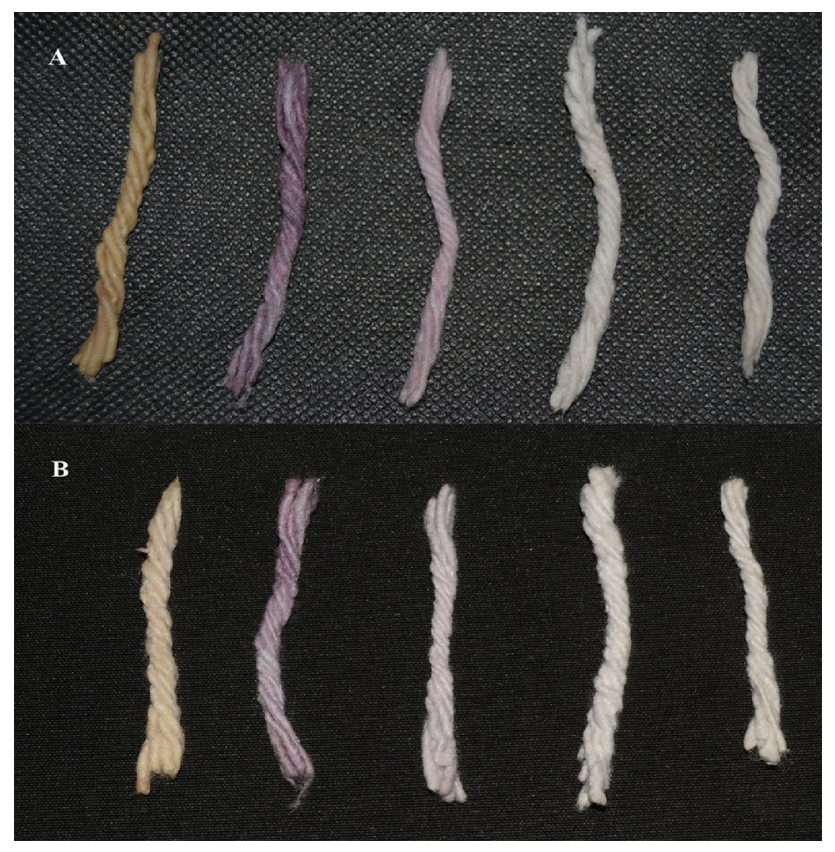

Figure 9. (A) cotton material dyed using gold nanoparticle colloid solution, (B) cotton material after exposure to various stress conditions.

12, 17-Trinaphthylenetetrone (12.658\%) major peak area. The compound names, molecular weight, empirical formula, retention time, peak area percentages, and structure of the identified compounds are given in Table 2.

Dye fixing. Synthesis of various coloured gold nanoparticles. The variety of attractive colors will be produced by altering the concentration of the gold ion solution as $10^{-2}, 10^{-3}, 10^{-4}, 10^{-5}$, and $10^{-6} \mathrm{M}$ solutions. They exhibit reddish-brown, purple, pink, pale yellow and colorless solutions respectively containing nanoparticles (Fig. 7). The particles exhibit different colors due to surface plasmon resonance effects in the visible region with the collective plasmon oscillations at the metal surface ${ }^{40}$. The rising of colors was based on the size of the gold nanoparticles. The colors of the cotton wicks were mainly various shades of brown, blue-purple, and shade blue colored fabrics produced.

Dyeing of cotton wicks. The different colored gold nanoparticles can be readily attached to cotton wicks. Figure 8 shows the cotton wicks before and after the dyeing process. Purple and red-colored gold nanoparticle colloids were successfully attached with cotton wicks samples.

Colour fastness. Generally, some colors were degraded while exposed to UV light. So, the color fastness determines the quality of fibers ${ }^{4}$. Figure 9 shows the stable color of cotton wicks treated with gold nanoparticles after exposure to various conditions like acid, alkali, commercial detergent, and direct sunlight. The nanoparticles are embedded in the interspaces between the cross-linked fabric materials. This result indicates the dye is attached to fabrics and showed positive colorfastness because most dyes were still in a negative phase for colorfastness. Hence these results show that the cotton wicks are colorfast and stable so would be a viable alternative to current synthetic dyes.

Scanning electron microscope. The SEM characterization technique is used to determine the morphology and physical properties of nanomaterials. The distribution of nanomaterials on the solid surface can also be attained using the SEM technique. SEM image (Fig. 10) visualizes the green synthesized gold nanoparticles which are deposited on the surface of cotton fabrics. Cotton fabrics are immobilized in gold nanoparticles prepared at different concentrations. Figure 10 shows the control and other concentrations used for coating the cotton fabrics with gold nanoparticle solution. The image depicts that many nanoparticles were adsorbed on the surface of the cotton fabrics with high aggregation. The particles are well dispersed and deposited on the surface of the cotton fabrics at $1 \mathrm{mM}$ concentration of gold nanoparticles solution and other concentrations show the low distribution of gold nanoparticles. Moreover, gold nanoparticles were not identified on the surface of cotton fabrics treated with other low concentrations $(0.1 \mathrm{mM}, 0.01 \mathrm{mM}$, and $0.001 \mathrm{mM})$. The low concentration of gold nanoparticle solutions does not influence the binding of nanoparticles on the surface of cotton fabrics. As such, $1 \mathrm{mM}$ gold nanoparticle solution was chosen as good results of our studies of immobilization of cotton fabrics. 


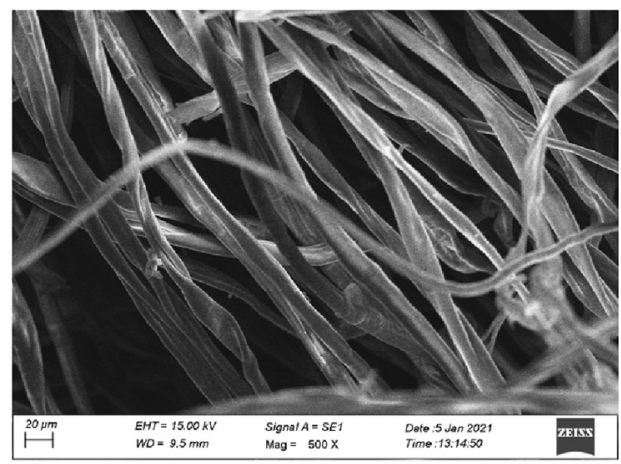

Control

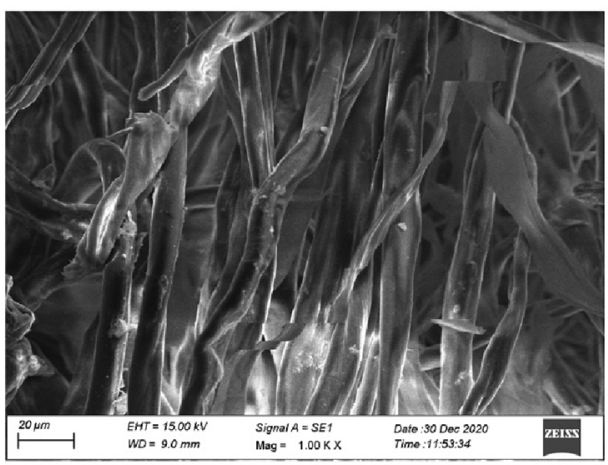

$1 \mathrm{mM}$

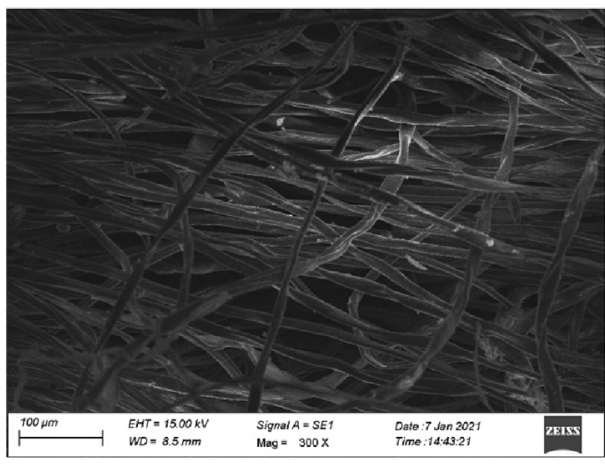

$0.01 \mathrm{mM}$
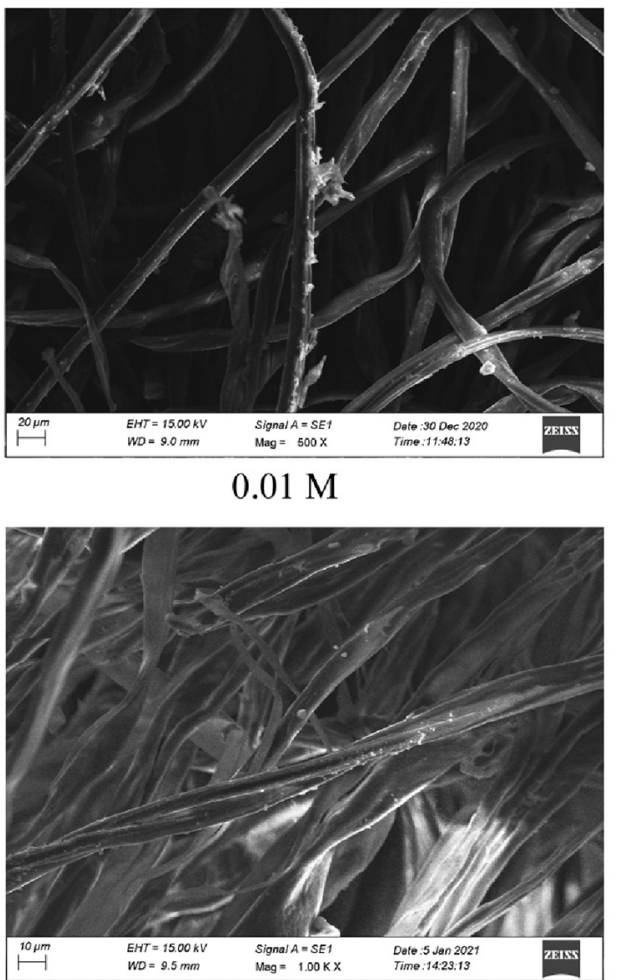

$0.1 \mathrm{mM}$

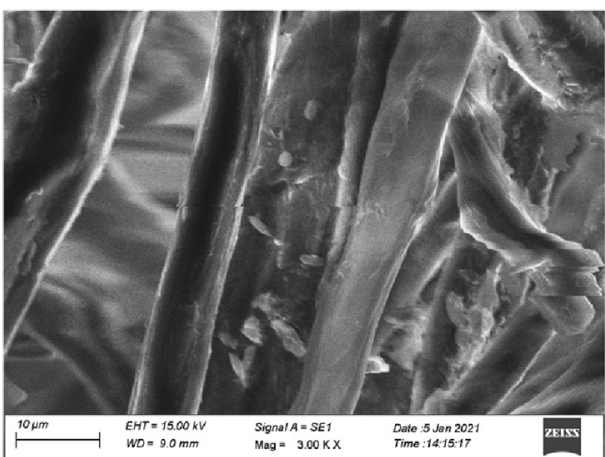

$0.001 \mathrm{mM}$

Figure 10. SEM image of gold nanoparticle dyed cotton material at various concentrations.

\section{Conclusion}

Gold nanoparticles impart different colors depending on their (Localized Surface Plasmon Resonance) LSPR in solution. The colors imparted by the nanoparticle solution were employed to dye cotton fabrics and the stability of the dyes on the fixed fabric was tested. These tests showed a positive result in the dyeing process. In summary, this paper presents an innovative approach to the synthesis and use of gold nanoparticles as novel stable colorants utilizing the surface plasmon resonance coloring effect of nanogold.

Received: 30 July 2020; Accepted: 25 May 2021

Published online: 24 June 2021

\section{References}

1. Morton, J. F. Fruits of Warm Climates 304 (Creative Resource Systems Inc., 1987).

2. Pedraza-Chaverri, J., Cárdenas-Rodríguez, N., Orozco-Ibarra, M. \& Pérez-Rojas, J. M. Medicinal properties of mangosteen (Garcinia mangostana). Food Chem. Toxicol. 46, 3227 (2008).

3. Zadernowski, R., Czaplicki, S. \& Naczk, M. Phenolic acid profiles of mangosteen fruits (Garcinia mangostana). Food Chem. 112, 685 (2009)

4. Sen, A. K. et al. A xanthone from Garcinia mangostana. Phytochemistry 19, 2223 (1980).

5. Balasubramanian, K. \& Rajagopalan, K. Novel xanthones from Garcinia mangostana, structures of BR-xanthone-A and BRxanthone-B. Phytochemistry 27, 1552 (1988). 
6. Hebeish, A. et al. Highly effective antibacterial textiles containing green synthesized silver nanoparticles. Carbohydr. Polym. 86, $936(2011)$.

7. Liu, X., Atwater, M., Wang, J. \& Huo, Q. Extinction coefficient of gold nanoparticles with different sizes and different capping ligands. Colloids Surf. B: Biointerfaces. 58, 3 (2007).

8. Philip, D. Biosynthesis of Au, Ag and Au-Ag nanoparticles using edible mushroom extract. Spectrochim. Acta A 73, 374 (2009).

9. Zheng, B. et al. Preparation of gold nanoparticles on eggshell membrane and their biosensing application. Talanta 82, 177 (2010).

10. Rajathi, F. A. A., Parthiban, C., Kumar, V. G. \& Anantharaman, P. Biosynthesis of antibacterial gold nanoparticles using brown alga, Stoechospermum marginatum (kützing). Spectrochim. Acta A 2, 99 (2012).

11. Muthuvel, A., Adavallan, K., Balamurugan, K. \& Krishnakumar, N. Biosynthesis of gold nanoparticles using Solanum nigrum leaf extract and screening their free radical scavenging and antibacterial properties. Biomed. Prev. Nutr. 4, 325 (2014).

12. Tripathi, R. M., Shrivastav, B. R. \& Shrivastav, A. Antibacterial and catalytic activity of biogenic gold nanoparticles synthesised by Trichoderma harzianum. IET Nanobiotechnol. 12, 509 (2018).

13. Kuppusamy, P. et al. Intracellular biosynthesis of Au and Ag nanoparticles using ethanolic extract of Brassica oleracea L. and studies on their physicochemical and biological properties. J. Environ. Sci. 29, 151 (2015).

14. Mishra, P. et al. Facile bio-synthesis of gold nanoparticles by using extract of Hibiscus sabdariffa and evaluation of its cytotoxicity against U87 glioblastoma cells under hyperglycemic condition. Biochem. Eng. J. 105, 264 (2016).

15. Sathishkumar, G. et al. Cannonball fruit (Couroupita guianensis, Aubl.) extract mediated synthesis of gold nanoparticles and evaluation of its antioxidant activity. J. Mol. Liq. 215, 229 (2016).

16. Irfan, M., Ahmad, T., Moniruzzaman, M. M., Abdullah, B. B. \& Bhattacharjee, S. Ionic liquid mediated biosynthesis of gold nanoparticles using Elaeis guineensis (oil palm) leaves extract. Procedia Eng. 148, 568 (2016).

17. Hoshyar, R., Khayati, G. R., Poorgholami, M. \& Kaykhaii, M. A novel green one-step synthesis of gold nanoparticles using crocin and their anti-cancer activities. J. Photochem. Photobiol. B 159, 237 (2016).

18. Johnston, J. H. \& Lucas, K. A. The use of dual reductants in gold nanoparticle syntheses. Gold Bull. 44, 85 (2011).

19. Tang, B. et al. Functionalization of bamboo pulp fabrics with noble metal nanoparticles. Dyes Pigm. 113, 289 (2015).

20. Cao, X. et al. Highly sensitive detection of melamine based on fluorescence resonance energy transfer between rhodamine B and gold nanoparticles. Dyes Pigm. 111, 99 (2014).

21. Bennur, T., Khan, Z., Kshirsagar, R., Javdekar, V. \& Zinjarde, S. Biogenic gold nanoparticles from the Actinomycete Gordonia amarae: application in rapid sensing of copper ions. Sens. Actuat. B: Chem. 233, 684 (2016).

22. Blosi, M., Albonetti, S., Gatti, F., Baldi, G. \& Dondi, M. Au-Ag nanoparticles as red pigment in ceramic inks for digital decoration. Dyes Pigm. 94, 355 (2012).

23. Mata, R., Bhaskaran, A. \& Sadras, S. R. Green-synthesized gold nanoparticles from Plumeria alba flower extract to augment catalytic degradation of organic dyes and inhibit bacterial growth. Particuology 24, 78 (2016).

24. Sinha, T. \& Ahmaruzzaman, M. High-value utilization of egg shell to synthesize silver and gold-silver core shell nanoparticles and their application for the degradation of hazardous dyes from aqueous phase-A green approach. J. Colloid Interf. Sci. 453, 115 (2015).

25. Tang, B. et al. Application of anisotropic silver nanoparticles: multifunctionalization of wool fabric. J. Colloid Interf. Sci. 356, 513 (2011).

26. Dong, H. \& Hinestroza, J. P. Metal nanoparticles on natural cellulose fibers: electrostatic assembly and in situ synthesis. ACS Appl. Mat. Interfaces 1, 797 (2009).

27. Torkian, L., Radmanesh, E. \& Daghighi, M. Effects of starch addition on the characteristics of CoAl2O4 nano pigments. Colour. Technol. 130, 376 (2014).

28. Afshari, S., Montazer, M., Harifi, T. \& Mahmoudi Rad, M. A coloured polyester fabric with antimicrobial properties conferred by copper nanoparticles. Colour. Technol. 135, 427 (2019).

29. Chattopadhyyay, D. P. \& Patel, B. H. Improvement in physical and dyeing properties of natural fibres through pre-treatment with silver nanoparticles. Indian J. Fibre Text. Res. 34, 368 (2009).

30. Singaravelu, G., Arockiamary, J. S., Kumar, V. G. \& Govindaraju, K. A novel extracellular synthesis of monodisperse gold nanoparticles using marine alga, Sargassum wightii Greville. Colloid Surf. B: Biointerfaces 57, 97 (2007).

31. Inbakandan, D., Venkatesan, R. \& Ajmal Khan, S. Biosynthesis of gold nanoparticles utilizing marine sponge Acanthella elongata (Dendy, 1905). Colloid Surf. B Biointerfaces 81, 634 (2010).

32. Prasad, T. N. V. K. V. \& Elumalai, E. K. Biofabrication of Ag nanoparticles using Moringa oleifera leaf extract and their antimicrobial activity. Asian Pac. J. Trop. Biomed. 1, 439 (2011).

33. Kamat, P. V., Flumiani, M. \& Hartland, G. V. Picosecond dynamics of silver nanoclusters: photoejection of electrons and fragmentation. J. Phys. Chem. B. 102, 3123 (1998).

34. Rani, P. U. \& Rajasekharreddy, P. Green synthesis of silver-protein (core-shell) nanoparticles using Piper betle L. leaf extract and its ecotoxicological studies on Daphnia magna. Colloids Surf A. 389, 188 (2011).

35. Kumar, R., Roopan, S. M., Prabhakarn, A., Khanna, V. G. \& Chakroborty, S. Agricultural waste Annona squamosa peel extract: biosynthesis of silver nanoparticles. Spectrochim Acta Part A. 90, 173 (2012).

36. Borchert, H. et al. Determination of nanocrystal sizes: a comparison of TEM, SAXS, and XRD studies of highly monodisperse CoPt3 particles. Langmuir 21, 1931 (2005).

37. Shukla, V. K., Singh, R. P. \& Pandey, A. C. Water purification by polymer nanocomposites: an overview. J. Alloys Comps. 207, L13 (2010).

38. Deshpande, R. et al. Rapid biosynthesis of irregular shaped gold nanoparticles from macerated aqueous extracellular dried clove buds (Syzygium aromaticum) solution. Colloid Surf. B: Biointerfaces 79, 235 (2010).

39. Chen, F., Wang, Y., Ma, J. \& Yang, G. A biocompatible synthesis of gold nanoparticles by Tris (hydroxymethyl) aminomethane. Nanoscale Res. Lett. 9, 220 (2014).

40. Mulvaney, P. Surface plasmon spectroscopy of nanosized metal particles. Langmuir 12, 788 (1996).

41. Johnston, J. H., Richardson, M. J. \& Burridge, K. A. Engineered nanomaterials: Scope in today's textile industry. NSTI-Nanotech. $1,712(2008)$.

\section{Acknowledgements}

The authors thank Dr.Vijayakumar, VIT-SIF Lab, SAS, Chemistry Division for NMR and GC-MS Analysis for Gas Chromatography-Mass Spectrometry analysis.

\section{Author contributions}

M.S. and M.V. have done all laboratory works and prepared manuscript and related works. G.A. have reviewed and made corrections for the manuscript. All authors reviewed the manuscript.

\section{Competing interests}

The authors declare no competing interests. 


\section{Additional information}

Correspondence and requests for materials should be addressed to M.S.

Reprints and permissions information is available at www.nature.com/reprints.

Publisher's note Springer Nature remains neutral with regard to jurisdictional claims in published maps and institutional affiliations.

(c) (1) Open Access This article is licensed under a Creative Commons Attribution 4.0 International License, which permits use, sharing, adaptation, distribution and reproduction in any medium or format, as long as you give appropriate credit to the original author(s) and the source, provide a link to the Creative Commons licence, and indicate if changes were made. The images or other third party material in this article are included in the article's Creative Commons licence, unless indicated otherwise in a credit line to the material. If material is not included in the article's Creative Commons licence and your intended use is not permitted by statutory regulation or exceeds the permitted use, you will need to obtain permission directly from the copyright holder. To view a copy of this licence, visit http://creativecommons.org/licenses/by/4.0/.

(C) The Author(s) 2021 